\title{
BIOMECÂNICA DO MOVIMENTO HUMANO
}

\author{
BIOMECHANICS OF HUMAN MOVEMENT
}

\section{BIOMECÁNICA DEL MOVIMIENTO HUMANO}

\author{
Bruno Mezêncio \\ https://orcid.org/0000-0001-6293-5571 (iD \\ http://lattes.cnpq.br/9793275013451101 \\ Universidade de São Paulo (São Paulo, SP - Brasil) \\ mezencio@usp.br \\ Jacielle Carolina Ferreira \\ https://orcid.org/0000-0002-9321-749X (iD \\ http://lattes.cnpq.br/4097604888377977 \\ Universidade Federal de Mato Grosso (Cuiabá, MT - Brasil) \\ jacielle@ufmt.br \\ Alberto Carlos Amadio \\ https://orcid.org/0000-0002-2527-2163 (iD \\ http://lattes.cnpq.br/0634221030546752 \\ Universidade de São Paulo (São Paulo, SP - Brasil) \\ acamadio@usp.br
}

\begin{abstract}
Resumo
A Biomecânica é uma ciência do grupo das ciências biológicas que trata de análises físico-matemáticas de sistemas biológicos e, como consequência, de movimentos humanos. Estes movimentos são analisados através de leis e normas mecânicas com relação a parâmetros específicos do sistema biológico. Atualmente a Biomecânica constituise numa disciplina curricular presente na quase totalidade dos cursos universitários de formação de bacharéis e licenciados em Educação física e Esporte, Fisioterapia, entre outros cursos. Sua consolidação foi acompanhada por uma grande evolução nas técnicas e recursos metodológicos aplicados, e ainda permanece em amplo desenvolvimento. Pretende-se com este artigo, apresentar um breve histórico da biomecânica como área de investigação, descrever seus principais métodos de investigação, sumarizar as principais particularidades da biomecânica no meio liquido e apresentar as atuais tendências e perspectivas futuras para a investigação do movimento humano.
\end{abstract}

Palavras-chave: Cinemetria; Dinamometria; Eletromiografia; Meio Líquido.

\begin{abstract}
Biomechanics is a science of the biological sciences group that deals with physical-mathematical analysis of biological systems and, as a consequence, of human movements. These movements are analyzed through mechanical laws and regulations regarding specific parameters of the biological system. Currently, Biomechanics is a curricular subject present in almost all university courses for the formation of graduates in Physical Education and Sport, Physiotherapy, among other courses. Its consolidation was accompanied by a great evolution in the techniques and methodological resources applied, and it still remains in wide development. The aim of this article is to present a brief history of biomechanics as an area of investigation, describe its main research methods, summarize the main characteristics of biomechanics in the liquid medium and present current trends and future perspectives for the investigation of human movement.
\end{abstract}

Keywords: Kinemetry; Dynamometry; Electromyography; Liquid Medium.

\section{Resumen}

La biomecánica es una ciencia del grupo de las ciencias biológicas que se ocupa del análisis físico-matemático de los sistemas biológicos y, como consecuencia, de los movimientos humanos. Estos movimientos se analizan 
mediante leyes y regulaciones mecánicas sobre parámetros específicos del sistema biológico. Actualmente, la Biomecánica es una asignatura curricular presente en casi todos los cursos universitarios para la formación de licenciados y graduados en Educación Física y Deporte, Fisioterapia, entre otros cursos. Su consolidación estuvo acompañada de una gran evolución en las técnicas y recursos metodológicos aplicados, y aún permanece en amplio desarrollo. El objetivo de este artículo es presentar una breve historia de la biomecánica como área de investigación, describir sus principales métodos de investigación, resumir las principales características de la biomecánica en el medio líquido y presentar las tendencias actuales y perspectivas de futuro para la investigación del movimiento humano.

Palabras clave: Cinemetría; Dinamometría; Electromiografía; Medio Líquido.

\section{INTRODUÇÃO}

Biomecânica é uma disciplina, entre as ciências derivadas das ciências naturais, que se ocupa com análises físicas de sistemas biológicos, consequentemente análises físicas de movimentos do corpo humano (AMADIO et al., 1999; BAUMANN, 1992). Estes movimentos são estudados através de leis e padrões mecânicos em função das características especificas do sistema biológico humano, incluindo conhecimentos anatômicos e fisiológicos. Quando dimensionamos a biomecânica no contexto das ciências derivadas, cujo objetivo é estudar o movimento humano, esta contextualização científica apoia-se em dois fatos fundamentais: a) a biomecânica apresenta claramente definido seu objeto de estudo, definindo assim sua estrutura de base do conhecimento; b) seus resultados de investigações são obtidos através do uso de métodos científicos (AMADIO, 1989).

Portanto a Biomecânica é uma ciência do grupo das ciências biológicas que trata de análises físico-matemáticas de sistemas biológicos e, como consequência, de movimentos humanos. Estes movimentos são analisados através de leis e normas mecânicas com relação a parâmetros específicos do sistema biológico. Por motivos teórico-metodológicos, os aspectos mecânicos e biológicos podem ser tratados separadamente na biomecânica, sem que se esqueça que a análise de movimentos ocupa-se de movimentos complexos de organismos biológicos. Tem ainda uma grande importância, no que diz respeito a relação entre a física e a biologia, as transformações e modificações das leis mecânicas pelas condições e influências biológicas. Em situações específicas, quando as interações do corpo humano com um meio fluído são relevantes para análises desejadas um ramo especifico da física denominado mecânica dos fluídos também se faz necessário.

Segundo Blaser (1971), a biomecânica é uma extensão das disciplinas físicas em fenômenos de sistemas biológicos com suas particularidades. Em princípio deve-se considerar que a estrutura funcional de um sistema biológico passou por um processo organizacional evolutivo de otimização, que se diferencia sensivelmente do caminho de aperfeiçoamento 
técnico do movimento. Em contraposição a um corpo rígido, a estrutura biológica do corpo humano permite a produção de forças através da contração muscular, que transforma o corpo num sistema independente e assim acontece o movimento.

A biomecânica está fortemente ligada a mecânica, que por sua vez é um ramo da física aplicada, que estuda a ação de forças e seus efeitos. Portanto, ao interpretar a biomecânica, devemos também entender o significado associado da mecânica. Neste contexto é que Hatze (1974) distingue Biocinemática, Biodinâmica e Bioestática, ou seja, o autor discute que a utilização do termo biomecânica sofre restrições em função do conceito definido a partir do termo mecânica. A Física - mecânica, ocupa-se portanto, sobretudo com a redução de achados ou descobertas naturais com a sua causa e relação de dependência de efeito e desta forma a determinação de procedimentos estruturados em forma de princípios e leis que permitem o prognóstico de fenômenos com consequentes representações e formulações matemáticas.

Conhecimento científico possibilita o desenvolvimento de métodos para o estudo de fenômenos naturais, indispensáveis para a compreensão dos parâmetros que compõem o universo do movimento humano. Ainda através da ciência, observamos não só a perfeita correlação entre os fenômenos físicos, mas também as relações entre suas causas e efeitos, evidenciando ser possível a alteração e ou modificações na estrutura de movimento, através de bases físico-matemáticas. Temos ainda a acrescentar que em função da preocupação em estudar estruturas e sistemas biológicos, hoje, neste universo das biociências, a biomecânica apresenta-se com um significado e área de atuação ainda mais amplo do que se questionássemos apenas em função da mecânica entre suas bases de estudo, caracterizandose, portanto, seu domínio de estudos no contexto da Biofísica. Assim interpreta-se a biomecânica como sendo oriunda da Biofísica, ou seja, é o estudo da estrutura e da função dos sistemas biológicos utilizando métodos da mecânica. Glaser (GLASER, 2012), caracteriza a Biofísica no domínio de conhecimentos com o objetivo de estudar os fenômenos físicobiológicos, portanto que envolvem organismos vivos, ou seja, o estudo da biologia valendose de métodos e princípios científicos da física.

O movimento pode ser conceituado a partir de um sistema de comportamento físico marcado por normas, regras e convenções, cujo referencial se assenta em respostas anatômicas, fisiológicas e biomecânicas do corpo humano. Em contraposição ao corpo rígido, como definido na mecânica clássica, a estrutura biológica do corpo humano permite a 
produção de forças internas por meio da contração muscular, que transforma o corpo em um sistema autônomo e independente e assim acontece o movimento (AMADIO et al., 1999), entendido a partir de simplificações pelo modelo das relações tipo causa-efeito. Deve-se considerar ainda, que a estrutura funcional do sistema biológico humano passou por um processo de desenvolvimento evolutivo e se diferencia sensivelmente do caminho de aperfeiçoamento técnico do movimento.

O atual desenvolvimento da Biomecânica do esporte, no contexto da moderna Ciência do esporte, se expressa pelos novos procedimentos e técnicas de investigação, nas quais podemos reconhecer a tendência crescente de se combinar várias disciplinas científicas na análise do movimento esportivo. Nos últimos anos o progresso das técnicas de medição, armazenamento e processamento de dados contribuem enormemente para a análise do movimento esportivo. É claro que nenhuma disciplina se desenvolve por si mesma, ou seja, a Biomecânica recorre a um complexo de disciplinas científicas, e, particularmente na Biomecânica do esporte pode-se observar uma estreita relação de dependências entre as necessidades e exigências da prática esportiva.

A biomecânica configura-se desta forma como uma disciplina com forte característica multidisciplinar, cuja meta central é a análise dos parâmetros físicos do movimento, em função das características anatômicas e fisiológicas do corpo humano e de restritores ambientais. A análise biomecânica do movimento humano é baseada em seus quatro principais métodos de investigação: a Cinemetria, a Dinamometria, a Eletromiografia e a Antropometria (AMADIO, 2000). Pretende-se com este artigo, portanto, apresentar um breve histórico da biomecânica como área de investigação, descrever seus principais métodos de investigação, sumarizar as principais particularidades da biomecânica no meio liquido e apresentar as atuais tendências e perspectivas futuras para a investigação do movimento humano.

\section{HISTÓRICO DA BIOMECÂNICA NO BRASIL}

A preocupação com a análise física do movimento humano é antiga. Obras clássicas de pensadores como Aristóteles evidenciam que o interesse do homem em analisar o movimento, a partir de preceitos físicos, é muito antigo. Interesse esse que se aprofundou durante os séculos seguintes, como demonstram os estudos clássicos de Borelli (século XVI) e Marey (século XIX), e que continua em curso até os dias atuais. Entretanto, apesar de o estudo 
do movimento ser antigo, a consolidação da Biomecânica como uma ciência e, posteriormente, como uma disciplina acadêmica, é recente (AMADIO; SERRÃO, 2011).

Quanto aos aspectos históricos do desenvolvimento da biomecânica, gostaríamos apenas de registrar um fato que marcou o início da atuação e coordenação internacional da Biomecânica do esporte, que começou, segundo Hochmuth (1974), com um Encontro Internacional relativo a perguntas fundamentais do papel da biomecânica para movimentos esportivos, em 1960, na cidade de Leipzig - Alemanha Oriental, e através da UNESCO que deu suporte e estrutura para o primeiro Seminário Internacional de Biomecânica, em 1969, na cidade de Zuerich - Suiça.

Portanto, a evolução continuou e a ótica pela qual os estudos se desenvolviam se viu ampliada. Isto ficou marcado, principalmente, pela inclusão dos princípios e leis físicomatemáticos nos métodos de investigação. Assim, ao que tudo indica foi Borelli, com a publicação de seu tratado "De Motu Animalium"(BORELLI, 1685), que deu os primeiros passos na direção das ampliações conceituais e instrumentais que nos remetem à biomecânica atual muito embora sofra críticas pelo trabalho que desenvolveu acerca da contração muscular, Borelli é considerado o pai da moderna biomecânica do aparelho locomotor.

No que diz respeito à Biomecânica do esporte há registros indicando que antes mesmo do início do século XX estudos com preocupações extremamente semelhantes a determinados setores da Biomecânica do esporte atual, como é o caso do estudo sobre tênis publicado pela Transactions of the Royal Society no ano de 1893, já vinham sendo realizados e publicados, em acordo com (BATISTA; AMADIO, 2005).

Outro fato importante, ainda segundo os supra referidos Autores, marca a história da biomecânica no cenário da Educação física brasileira, e que também poderia ter sido um marco no cenário mundial se ocorrido em outro contexto, deu-se em 1963, quando o engenheiro naval Ary Biolchini proferiu uma conferência intitulada "A Natação vista por um Engenheiro", no curso de extensão universitária em Cinesiologia da Natação, da Escola de Educação Física da Universidade do Brasil (hoje Universidade Federal do Rio de Janeiro -UFRJ). Em uma ação precursora, no contexto da Biomecânica do esporte, o engenheiro Biolchini apresentou hipóteses acerca da mecânica natatória, as quais só seriam abordadas em congresso, para o público mundial, em 1970 pelo pesquisador norte americano James Counsilman. Os trabalhos apresentados por este autor fazem parte do que poderia ser denominado pedra fundamental no estudo da biomecânica da natação. 
Registro digno de nota neste contexto foi a publicação do livro "Natação" (Editora Melhoramentos, Rio de Janeiro), escrito pela Professora MARIA LENK em 1942 onde apresenta um capítulo, "Ciência e Natação" e refere-se à descrição analítica dos movimentos a fim de esclarecer as dúvidas de cuja solução depende o êxito do nadador. Aborda tópicos descritos como Cinesiológicos e Físicos. Descreve a Autora que na análise do nado o que mais de perto interessou-a foi a apresentação do Butterfly pois, ela própria, em função dos conhecimentos desenvolvidos, aplicou na sua prática de treinamento como atleta, os ensinamentos de forma muito diferente daquela que os campeões americanos para o nado peito apregoavam. Declara ainda Maria Lenk que a eficiência já provada é evidenciada neste livro de forma teórica e constitui-se como o primeiro livro escrito em idioma português sobre biomecânica da natação (LENK, 1942). Importante registrar que Maria Emma Hulda Lenk Ziegler (1915-2007) foi Professora Catedrática da Universidade do Brasil, como atleta foi campeã e recordista mundial de 200 e 400m., condecorada pelo Comitê Olímpico Internacional com a Ordem Olímpica e licenciada em Educação Física pela Universidade de São Paulo, em 1935.

A história da biomecânica no Brasil, começou a ser escrita há poucos anos. Esta trajetória foi fortemente influenciada pelo apoio que algumas instituições de ensino superior brasileiras receberam do governo alemão, ou seja, em acordo com os registros disponíveis, a efetiva introdução da Biomecânica nos cursos de Graduação e Pós-graduação em Educação física no Brasil teve impulso na época em que se estava concretizando o acordo cultural de cooperação entre o Brasil e a República Federal da Alemanha, iniciado por volta dos anos de 1960. Um dos marcos históricos desta relação deu-se em 1965, ano em que foi concretizado o convênio cultural de cooperação entre o Brasil e a República Federal da Alemanha para a introdução da biomecânica nos cursos de Educação física no Brasil (DIEM; LÖCKEN; HUMMEL, 1983). Como uma das ações previstas nesse convênio, no ano de 1976, o Prof. Dr. Hartmut Riehle ministrou cursos na Escola de Educação Física da Universidade de São Paulo e na Universidade Federal de Santa Maria, com o intuito de fomentar o desenvolvimento da área, e estabelecer as bases para o curso de formação de especialistas em biomecânica. A partir do impulso oferecido pelo convênio cultural Brasil \& Alemanha, observou-se um expressivo aumento no número de pesquisadores dedicados ao estudo acadêmico das questões biomecânicas.

Ainda conforme o programa do acordo de cooperação Brasil \& Alemanha, em 1979, o Prof. Dr. Wolfgang Baumann (Institut für Biomechanik da Deutsche Sporthochschule 
Köln - República Federal da Alemanha) esteve no Brasil com o propósito de visitar as seguintes universidades: Universidade de São Paulo, Universidade Federal de Santa Maria, Universidade Federal do Rio Grande do Sul, Universidade Federal do Rio de Janeiro e Universidade Estadual do Rio de Janeiro. Estas visitas tinham como objetivos diagnosticar a situação daquelas Universidades, estimar a possibilidade das mesmas desenvolverem projetos em biomecânica e também fornecer orientação especializada a fim de serem construídos ou ampliados os laboratórios dos departamentos que pudessem desenvolver esse trabalho. Estabeleceu-se a perspectiva para que esse trabalho trouxesse reflexos tanto para a formação de professores quanto nas atividades de pesquisa daqueles centros selecionados (DIEM; LÖCKEN; HUMMEL, 1983).

Tal condição levou a biomecânica a se expandir para além do espaço disciplinar da Educação física e do Esporte, gerando importantes relações multidisciplinares. Como evidência dessa expansão deve-se citar a estruturação dos primeiros encontros científicos brasileiros destinados à discussão da biomecânica, merece destaque, por se tratar do primeiro evento acadêmico da biomecânica brasileira, a realização do "I Encontro Nacional de Docentes de Cinesiologia e Biomecânica", em 1988 organizado pela Escola Superior de Educação Física da Universidade Federal do Rio Grande do Sul. Neste encontro participou, como conferencista convidado do exterior, Prof. Dr. Wolfgang Baumann do Institut für Biomechanik - Deutsche Sporthochschule Köln, Alemanha (AMADIO; SERRÃO, 2011).

Desde 1980 a biomecânica vinha passando por um expressivo crescimento e expansão do campo de aplicação, no mundo todo e, também no Brasil. Durante algum tempo percebeu-se uma implementação lenta, porém desde que se iniciou no Brasil, no ano de 1988, a referida reunião de estudiosos e interessados em trocar experiências, a taxa de crescimento aumentou expressivamente. Uma das consequências deste crescimento tem sido a ampliação da biomecânica para além do espaço da Educação física e Esporte. Em sua terceira edição, o encontro ocorreu em Ouro Preto - MG, no ano de 1991, promovido pela Universidade Federal de Ouro Preto, quando o encontro passou a ser denominado "Congresso Nacional de Biomecânica". Foi durante a quarta edição deste evento, realizada em São Paulo - SP, promovido pela Escola de Educação Física e Esporte da Universidade de São Paulo, em dezembro de 1992, é que foi fundada, em Assembleia Geral, a Sociedade Brasileira de Biomecânica - SBB. A partir do quinto encontro, realizado em Campinas - SP, promovido pela Faculdade de Educação Física da Universidade Estadual de Campinas, no ano de 1993, o 
Congresso Nacional de Biomecânica passou a ser denominado "Congresso Brasileiro de Biomecânica" - CBB. Desde então o CBB é realizado bienalmente, sob a tutela e responsabilidade da SBB. A SBB foi criada com o objetivo de abrigar aqueles que demonstrassem interesse pela área, constituindo um quadro de afiliações por meio de seus associados e assumiu oficialmente a função de representante oficial da comunidade que atua nesse campo, respondendo pelo desenvolvimento da Biomecânica no Brasil.

A SBB constitui-se portanto numa sociedade civil de direito privado, sem fins lucrativos, que tem por objetivo reunir os profissionais da Biomecânica e ciências afins no território brasileiro, com as seguintes finalidades: promover e apoiar o aperfeiçoamento técnico e científico desses profissionais; estimular a criação de centros de pós-graduação através da colaboração com universidades e instituições de pesquisa; manter vinculação com entidades do país e do exterior, agindo como representante oficial da biomecânica brasileira; zelar pelos aspectos éticos do exercício da biomecânica; e organizar a realização do CBB e de outros eventos científicos, promovendo a divulgação de conhecimentos sobre a área (Sociedade Brasileira de Biomecânica, 2011). Criou-se mais tarde, a Revista Brasileira de Biomecânica - Brazilian Journal of Biomechanics (RBB-BJB) como sendo o órgão oficial representativo da SBB com a incumbência de promoção e divulgação científica da Biomecânica. Antes do seu lançamento, em novembro de 2000, os anais do CBB representavam o único meio de divulgação científica destinada exclusivamente à biomecânica existente no Brasil. Desde o seu lançamento, a RBB-BJB atua como o órgão de divulgação e promoção científica oficial da Biomecânica. Esta iniciativa teve o propósito de dotar a comunidade de um veículo de referência aos serviços, laboratórios e grupos de pesquisa (AMADIO; SERRÃO, 2012).

Desta maneira vimos a Biomecânica constituir-se numa disciplina curricular presente na quase totalidade dos cursos universitários de formação de bacharéis e licenciados em Educação física e Esporte, Fisioterapia, entre outros cursos. Vários laboratórios e núcleos de estudo estão se consolidando, assim como nos cursos de pós-graduação lato-sensu e stricto-sensu, observa-se a disciplina Biomecânica ocupar lugar de destaque. Assim percebemos que cada vez mais os profissionais de Educação física e Esporte têm-se atentado à importância dos conhecimentos que a Biomecânica oferece para a capacitação acadêmica e científica dos interessados (AMADIO et al., 1999).

\section{DESCRIÇÃO METODOLÓGICA DOS TESTES E MEDIDAS EM CINEMETRIA}


Dentre os métodos de investigação biomecânica, a cinemetria consiste no registro de imagens e reconstrução do movimento com auxílio de pontos específicos, baseados em modelos antropométricos, que estimam a localização dos pontos de interesse dos sujeitos avaliados. As imagens são registradas por câmeras e auxílio de software e hardware específicos. As coordenadas bi ou tridimensionais de cada ponto corporal para cada quadro, dentro do espectro da frequência de captura, são determinadas através desses marcadores utilizando algoritmos específicos.

A partir das trajetórias avaliadas ao longo do tempo, indicadores cinemáticos de importância estrutural para a avaliação do rendimento esportivo podem ser determinados, à saber: variações lineares e angulares de posição, velocidades lineares e angulares, velocidade do centro de gravidade, dos segmentos e das articulações, determinação das variações da aceleração do movimento, tempo de reação e tempo de movimento, entre outras (AMADIO; SERRÃO, 2011).

A aplicação de modelos do corpo humano nos dados cinemáticos obtidos permite a determinação de importantes propriedades do corpo analisado, como por exemplo a aplicação dos modelos de Hatze e Zatsiorsky (DE LEVA, 1996; HATZE, 1980), para a determinação das massas e propriedades inercias de cada segmento e de Hanavan (HANAVAN JÚNIOR, 1964) para a determinação da localização do centro de gravidade do corpo todo. Portanto as variáveis antropométricas atuam como auxiliares para a determinação de variáveis cinemáticas do corpo.

Para aplicações em cinemetria recomenda-se procedimentos e sistemas que utilizam câmeras digitais, com frequência do registro da imagem de acordo com a frequência natural do movimento a ser analisado e resolução espacial compatível com a acurácia mínima aceitável para a interpretação do movimento. Recomenda-se ainda que a propagação do erro de medida esteja abaixo de 5\% (AMADIO; SERRÃO, 2007).

Para a calibração das câmeras e, posterior reconstrução das coordenadas de pontos de interesse, recomenda-se a utilização do método DLT (Direct Linear Transformation) (ABDEL-AZIZ; KARARA, 2015), por tratar-se de procedimento padronizado, amplamente utilizado pela comunidade científica e de fácil aplicação. Com a popularização das câmeras com lentes do tipo "olhos de peixe", modelos de calibração com maior controle de distorções radiais foram desenvolvidos, os modelos de Zhang e Heikkila (HEIKKILA; SILVEN, 1975; ZHANG, 1999) são exemplos amplamente utilizados e de fácil aplicação. 
Os sistemas mais utilizados atualmente são sistemas ótico-eletrônicos que funcionam com utilização de marcadores ativos ou passivos e muitas vezes permitem a reconstrução em tempo real dos marcadores que são processados em alta frequência e resolução. Outros sistemas da determinação de variáveis cinemáticas são goniômetros, velocímetros, acelerômetros e centrais inercias. As centrais inerciais, também chamadas de IMU (Inertial Measurements Units) são compostas normalmente por um acelerômetro, um girometro e um magnetometro, e fornecem dados de variação da posição e orientação com 6 graus de liberdade. Com a utilização de procedimentos dinâmicos de calibração, as variáveis cinemáticas obtidas pelas IMUs podem ser relacionadas aos segmentos corporais ondes estas são fixadas (AHMAD et al., 2013; AMINIAN; NAJAFI, 2004). A vantagem da aplicação desses métodos em relação ao registro da imagem é a disponibilidade quase simultânea e direta dos resultados de medição além de uma menor limitação espacial do ambiente de coletas. Estes métodos também podem ser aplicados durante o treinamento técnico possibilitado feedback instantâneo, auxiliando na aprendizagem e aperfeiçoamento da técnica de movimento (AMADIO; SERRÃO, 2011).

\section{DESCRIÇÃO METODOLÓGICA DOS TESTES E MEDIDAS EM DINAMOMETRIA}

Dinamometria é o método utilizado para a avaliação da força na biomecânica. A definição do conceito de força, sob o aspecto físico, somente pode ser interpretada a partir do efeito de sua ação, e assim, podemos interpretar seus efeitos estático e dinâmico. A dinamometria engloba todos os tipos de medidas de força e pressão. As forças mensuráveis são as forças externas, transmitidas entre o corpo e o ambiente. (AMADIO; SERRÃO, 2007).

A descrição de aspectos biomecânicos do movimento humano está ligada às forças que causam o movimento observado, assim como suas repercussões no fenômeno analisado. A investigação da força de reação do solo (FRS) dos movimentos, bem como da distribuição de pressão dinâmica na superfície de contato, trazem importantes informações sobre a forma e características da sobrecarga mecânica sobre o aparelho locomotor humano, e é de fundamental importância para descrição do mesmo, pois ela reflete a somatória dos produtos da aceleração da massa de todos os segmentos do corpo (AMADIO; DUARTE, 1996). De particular interesse são as forças de reação do solo em atividades quasi-estáticas ou dinâmicas, pois juntamente com a constante peso corporal, essas forças de reação do solo são, 
geralmente, a causa de qualquer alteração do movimento do centro de gravidade do corpo (AMADIO; SERRÃO, 2007).

O instrumento básico em dinamometria é a plataforma de força, que mede fundamentalmente a FRS, seus momentos e o ponto de aplicação desta força. A plataforma de força fornece um sinal elétrico proporcional à força aplicada. Existem vários tipos de sensores para este tipo de medição sobressaindo-se os "strain gauges", piezoelétricos, piezoresistivos e capacitivos. Nas medições da FRS com a plataforma de força, são determinados três componentes de força e três componentes momentos atuante, com os quais duas coordenadas do ponto de aplicação da força podem ser determinadas definindo-se assim um sistema tridimensional de coordenadas onde as componentes de força podem ser observadas (AMADIO, 2000).

A FRS é representada em forma de vetores, em função da variável tempo, considerando-se a sua ação tridimensional (componente vertical, antero-posterior e médiolateral). Assim a plataforma quantifica a variação da FRS durante a fase de contato entre os corpos, quando ocorre a transferência destas forças externas para o corpo determinando alterações nas condições de movimento (AMADIO; SERRÃO, 2007).

Müller (1992) apresenta os principais procedimentos para medição de pressão plantar em biomecânica, baseado em registro da impressão, ópticos, acústicos, pneumáticos, hidráulicos, indutivos, piezoelétricos, capacitivos e resistivos. Ilustrando o quão diversificado é o desenvolvimento tecnológico quanto a sistemas e dispositivos utilizados para medição da distribuição da pressão plantar. O autor também avaliou a precisão de medidas ao utilizar diferentes sistemas de análise da distribuição da pressão plantar, comparado com as medidas

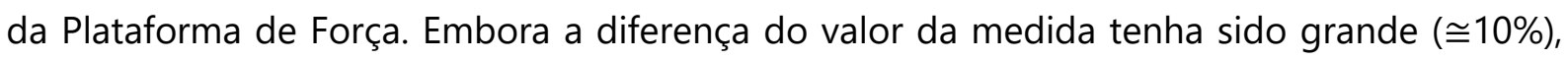
pode-se verificar, todavia uma concordância principal da forma das curvas para todos os testes.

Células de carga e sensores de torque como os utilizados em aparelhos isocinéticos também compõem os instrumentos que podem ser utilizados para avaliação das forças. Nestes casos a interação da força muscular do avaliado com o aparelho em si, e não com o solo é investigada, permitindo-se assim a obtenção de valores de força localizados ao invés de gerais.

\section{DESCRIÇÃO METODOLÓGICA DOS TESTES E MEDIDAS EM ELETROMIOGRAFIA}

Eletromiografia - EMG é o método de registro da atividade elétrica de um músculo, apresentando várias aplicações, por exemplo, o diagnóstico de doenças neuromusculares na 
clínica médica; biofeedback na reabilitação muscular; revela as ações musculares nos movimentos; entre outros (AMADIO; DUARTE, 1996). Na EMG de superfície a variação do potencial elétrico muscular, é medida nos eletrodos que são fixados na pele. É importante, portanto selecionar previamente os grupos musculares relevantes para o movimento analisado. Tradicionalmente o potencial de ação muscular é investigado paralelamente aos parâmetros mecânicos obtidos a partir da dinâmica e/ou cinemática, possibilitando uma visão da coordenação da atividade muscular (AMADIO; SERRÃO, 2007).

A EMG é um indicativo indireto que reflete os acontecimentos fisiológicos do músculo, e por isso os resultados são extremamente individualizados, sendo, portanto músculo e sujeito dependente. Por isso, é importante para EMG, que padrões para estes quesitos sejam propostos a fim de proporcionar a comparação mais fidedigna entre estudos semelhantes. A ISEK (International Society of Electrophysiology and Kinesiology) estabelece a padronização conceitual e protocolar para avaliações eletromiográficas. O projeto SENIAM (Surface EMG for a Non-invasive Assesment of Muscle) tem apresentado recomendações de configuração e posicionamento dos eletrodos (HERMENS et al., 2000).

Após a captação do sinal EMG este precisa ser analisado e interpretado. Existem dois tipos básicos de análises do sinal EMG: análise no domínio do tempo do sinal e análise no domínio da frequência do sinal. A análise no domínio do tempo do sinal permite principalmente a visualização do padrão de ativação muscular durante uma contração, podendo servir como referência para comparações entre diferentes tipos de contrações, exercícios e sobrecargas. A magnitude da ativação EMG também é uma importante variável a ser analisada no domínio do tempo, esta magnitude é estimada pela RMS (root mean square) do sinal, devido à elevada relevância do componente dinâmico associado a EMG. Este método permite ainda que relações entre força e atividade elétrica muscular possam ser observadas, apesar de ser uma vertente ainda controversa na literatura cientifica que aborda o assunto (STAUDENMANN et al., 2010). A análise no domínio da frequência permite identificar o espectro que frequência que compõe o sinal EMG. A principal variável analisada neste contexto é a mediana da frequência - MF que indica a frequência que corresponde à metade da energia do sinal e é utilizada principalmente em estudo visando à determinação da fadiga EMG ou em análises relacionadas à frequência de disparo (CIFREK et al., 2009; WAKELING, 2009). O comportamento esperado é que com o acumulo da fadiga haveria uma redução no valor da MF. 
Quanto ao tratamento dos sinais para as específicas situações de análise e interpretações recomenda-se rotinas e formalismos igualmente estabelecidos pela ISEK (MERLETTI; DI TORINO, 1999). Observa-se ainda haver duas formas principais de influenciar a fidelidade do sinal quando detectamos e registramos os sinais eletromiográficos. A primeira é a relação sinal/ruído, que é a razão entre a energia do sinal gerado pelo músculo e a energia do ruído incorporado ao sinal, definido como o conjunto dos sinais elétricos captados e que não fazem parte do sinal desejado, razão pela qual os procedimentos prévios de preparação da pele e posicionamento dos eletrodos é tão relevante. A segunda é a distorção do sinal devido ao próprio processamento pelo equipamento, significando uma alteração relativa em qualquer componente de frequência do sinal. A literatura aponta para uma faixa de frequências que vai de 0 a $500 \mathrm{~Hz}$, como sendo a faixa de energia utilizável do sinal EMG. Assim, a frequência de aquisição de dados eletromiográficos deve ser superior a $1000 \mathrm{~Hz}$, sendo utilizados filtros passa-alta entre 0 e $20 \mathrm{~Hz}$ e passa baixa entre 400 e $500 \mathrm{~Hz}$.

Outro aspecto importante da análise EMG é a normalização dos dados, que visa permitir a comparação de resultados de diferentes músculos, sujeitos e momentos de coleta. A normalização dos dados eletromiográficos pode ser elétrica (pelo próprio sinal EMG) ou mecânica (por variáveis como força e potência), as normalizações elétricas são largamente as mais utilizadas e, portanto as que serão aqui apresentadas. Dois aspectos são relevantes para se determinar o fator pelo qual o sinal deve ser normalizado: a tarefa a servir de referência e o tratamento aplicado aos dados desta. Com relação à tarefa o mais comum é a utilização de contrações isoladas (ou o mais próximo disso possível) dinâmicas ou isométricas, máximas ou submáximas, do músculo a ser normalizado. Outra possibilidade é a utilização de uma tarefa complexa como referência, por exemplo, um salto, ou um ciclo de passada na corrida, onde todos os músculos de interesse sejam solicitados. Dentre estas possibilidades aquela que é mais amplamente aplicada é a contração isométrica voluntária máxima para cada músculo a ser analisado. Após a seleção e registro da atividade EMG na tarefa de referência o sinal deve ser processado para que um valor de referência seja determinado. Usualmente o RMS do sinal é determinado em janelas entre 100 e 1000ms e o maior valor ou o valor médio de todas as janelas é utilizado para esta finalidade (BURDEN, 2010).

\section{BIOMECÂNICA DO MEIO LÍQUIDO}


Quando se analisa a interação entre os constituintes de um sistema biológico ou a interação do sistema biológico com um meio sólido a mecânica clássica, principalmente em sua formulação newtoniana, fornece um modelo físico praticamente completo que descreve com precisão o comportamento dos sistemas estudados pela biomecânica, já que o planeta pode ser considerado um referencial inercial para quase totalidade dos estudos biomecânicos. Esta formulação da mecânica clássica é conhecida desde 1687 com a publicação do Philosophiae naturalis principia mathematica por Isaac Newton. A mecânica dos fluidos por sua vez é muito mais recente e complexa. A mecânica dos fluidos moderna passou a ser reconhecida com tal apenas em 1904, com a formulação da camada limite, por Ludwig Prandtl (PRANDTL, 1904). Embora já houvesse estudos anterior sobre o comportamento dos fluidos (Arquimedes, Newton e Bernoulli, por exemplo), os modelos teóricos desenvolvidos até então nem sempre correspondiam aos resultados experimentais observados.

Um fluído é uma substância que se deforma continuamente quando submetido a uma tensão de cisalhamento de qualquer magnitude. A razão dos fluídos serem facilmente deformáveis está relacionada à sua estrutura molecular. Enquanto nos sólidos existe um pequeno espaçamento entre as moléculas e as forças intermoleculares são intensas, nos fluidos estas forças são fracas e existe um maior espaçamento entre as moléculas. Estas diferenças em nível molecular entre as substâncias também exige que a descrição do comportamento destas seja feito de modo diferenciado. A alta coesão das moléculas em um sólido faz com que o seu comportamento possa ser normalmente descrito em função de seu centro de massa, porém um fluido pode apresentar características diferentes em cada molécula. Como não é possível caracterizar cada molécula de um fluido individualmente, é necessário que se estabeleça uma quantidade de interesse, que é um volume com dimensões pequenas em relação ao sistema analisado e que deve conter uma grande quantidade de moléculas do fluido para que estas possam ser propriamente caracterizadas em função de seu comportamento médio. (MUNSON; YOUNG; OKIISHI, 2004).

Uma das propriedades que caracterizam um fluído é a sua massa específica ( $\rho$ ) que quantifica a massa do fluido por unidade de volume, esta propriedade descreve a inércia do fluido, ou seja, a dificuldade em se alterar seu estado de movimento. Considerando os efeitos da gravidade é possível calcular o peso específico de um fluído $(\gamma)$.

Outra propriedade inerente de um fluído é sua pressão (p), definida como a força normal por unidade de área exercida numa superfície plana (real ou imaginária) imersa no 
fluído. A pressão em um ponto é exercida em todas as direções e no caso da água, sua magnitude depende unicamente da profundidade do ponto em questão (z), já que a pressão existente no ponto é o resultado da força peso de toda coluna de água acima do ponto e da pressão atmosférica exercida na superfície livre (logo, $\left.p=p_{a t m}+\gamma z\right)$.

Quando um corpo se encontra completamente submerso na água, ele é submetido à força de pressão por todos os lados. Como a magnitude desta força é dependente unicamente da profundidade do ponto de interesse, a pressão exercida pelas moléculas de água dispostas lateralmente ao corpo são anuladas pelas moléculas contralaterais, que possuem pressões de mesma magnitude, porém com orientações opostas. No entanto as moléculas de água dispostas na face superior do corpo sempre possuem uma magnitude de pressão inferior àquelas dispostas na face inferior do corpo, por estarem mais próximas da superfície. Desta forma, este gradiente de pressões ao redor do corpo sempre produz uma resultante vertical orientada para cima, esta força resultante é chamada de empuxo, e seu módulo é proporcional ao peso do fluido deslocado pelo objeto, representado pelo produto do volume do objeto pela massa específica do fluido (ARCHIMEDES, 2009). Como as pressões atuam uniformemente ao redor do corpo, o empuxo resultante é aplicado no centro de volume do corpo, já a força peso do corpo, que possui orientação oposta a da força de empuxo, atua no centro de massa do corpo. No caso do centro de volume e do centro de massa do corpo analisado não serem coincidentes, a resultante das forças peso e de empuxo também produzira um torque sobre o corpo, proporcional à distancia horizontal entre estes dois pontos. Este torque produzirá um movimento de rotação no corpo até que os centros de volume e de massa do corpo estejam verticalmente alinhados (AMADIO; DUARTE, 1996).

Porém, este mecanismo de rotação do corpo só é relevante em condições estáticas, em situações dinâmicas, como na natação, as forças geradas por nadador e pelo escoamento são mais relevantes. A resistência que a água oferece ao deslocamento também possui um componente perpendicular ao deslocamento, esta força é chamada de sustentação, e atua verticalmente e para cima em todo o corpo; as forças propulsivas geradas pelo nadador também possuem componentes verticais, principalmente as geradas pelas pernas; além disso, os próprios movimentos de rotação de braços e pernas também geram momento angular que é transmitido para o corpo. É a somatória destas forças a principal causa o torque de rotação do corpo em uma condição dinâmica (YANAl; WILSON, 2008), em trabalhos experimentais de biomecânica este torque é comumente chamado de torque subaquático, que é definido como 
o produto entre a somatória das forças verticais no pé e a distancia entre o pé e o eixo de rotação do corpo, normalmente localizado no tórax, devido à alta flutuabilidade desta região do corpo causada pelos pulmões (PENDERGAST et al., 1977).

Considerando um escoamento sem influências externas e aplicando a $2^{\mathrm{a}}$ Lei de Newton às partículas fluídas, o seu deslocamento seria governado pelas forças peso e de pressão. Como a magnitude destas forças depende unicamente da profundidade, a força resultante em cada região do escoamento é constante, desta forma embora a aceleração e velocidade de uma partícula do fluído possam se alterar ao longo do escoamento, toda partícula que, em algum momento, ocupe aquela região do escoamento estará sujeita às mesmas forças. Este regime de escoamento é conhecido como regime permanente. As leis de Newton mostram que uma mudança de direção só é possível através da aplicação de uma força e é proporcional à magnitude desta. Assim conclui-se que toda partícula de fluído que cruza um mesmo ponto no espaço possui a mesma trajetória ao longo escoamento, já que está submetida às mesmas forças. Esta trajetória ao longo do escoamento é chamada de linha de corrente (MEZÊNCIO, 2017).

Daniel Bernoulli demonstrou que em um regime permanente, as propriedades de um escoamento se mantêm constantes dentro de uma linha de corrente para um fluido incompressível. Porém existe outra força que pode afetar as propriedades do escoamento, que é o atrito entre as próprias moléculas do fluído. Assim outro pressuposto assumido para equação de Bernoulli é que os efeitos viscosos do escoamento são negligenciáveis (BERNOULII; BERNOULLI, 1968).

O efeito do atrito entre as próprias moléculas do fluido é chamado de viscosidade. E é determinado pelo quanto ele se deforma para uma determinada tensão de cisalhamento. Devido às interações entre as moléculas, a tensão aplicada em uma determinada região é transmitida através do escoamento produzindo um gradiente de velocidades em função da distância entre as camadas do escoamento (du/dy). A lei da viscosidade de Newton estabelece que este gradiente de velocidade é proporcional à tensão de cisalhamento, esta constante de proporcionalidade é chamada de coeficiente de viscosidade dinâmico ( $\mu$ ) (MUNSON; YOUNG; OKIISHI, 2004).

A viscosidade de um escoamento também quantifica a capacidade do fluido de manter a estabilidade das linhas de corrente. Quando a viscosidade do fluido é suficiente, as linhas de corrente se mantém estáveis e paralelas umas as outras ao longo de todo o 
escoamento, este regime é conhecido como escoamento laminar. Quando a viscosidade do fluido não é suficiente para conter a energia cinética das linhas de correntes, elas podem perturbar umas as outras, causando mudanças bruscas no escoamento, gerando velocidade em sentido perpendicular ou até oposto à direção do escoamento, este regime é conhecido como escoamento turbulento. Neste regime de escoamento as linhas de corrente podem produzir um padrão típico, circular e estável chamado de vórtice (REYNOLDS, 1883; 1895).

Uma aplicação importante dos efeitos viscosos é vista no escoamento ao redor de um corpo submerso. O fluido em contato com uma superfície possui a mesma velocidade que ela, este fenômeno é chamado de condição de não escorregamento, isso faz que sempre haja um gradiente de velocidades no escoamento ao redor de um corpo submerso em um fluido (LAUGA; BRENNER; STONE, 2005). As partículas próximas ao corpo apresentam um gradiente de velocidades indo de zero, quando em contato com este, até a velocidade do escoamento, em uma região distante o suficiente do corpo para não sofrer os efeitos viscosos. Desta forma, este escoamento possui uma região próxima ao corpo submerso onde os efeitos viscosos são importantes, enquanto fora desta região estes efeitos são negligenciáveis, Esta região do escoamento onde as forças viscosas são importantes é chamada de camada limite (ANDERSON, 2005).

A força que atua nos corpos imersos num escoamento é resultado da interação entre o corpo e o fluido que o envolve. Estas forças podem ser normais à superfície do corpo devido ao efeito da pressão ou tangentes a ele devido ao efeito da tensão de cisalhamento causada pelos efeitos viscosos. A componente na direção do escoamento da resultante destas forças em toda a superfície do corpo imerso é o arrasto, e a componente perpendicular à direção do escoamento é a sustentação. Como é praticamente impossível conhecer as distribuições de tensão em um objeto, usualmente são utilizados coeficientes de arrasto e sustentação adimensionais obtidos experimentalmente. Como na água os efeitos inerciais $\left(\rho \sim 10^{3}\right)$ são normalmente mais relevantes que os viscosos $\left(\mu \sim 10^{-3}\right)$ as forças devido à pressão são predominantes em escoamentos sobre corpos imersos. O arrasto devido à tensão de cisalhamento e à pressão também é considerado conjuntamente, produzindo o coeficiente de arrasto total $(\mathrm{Cd})$. O coeficiente de arrasto é altamente dependente da forma do objeto e existem valores de coeficiente de arrasto determinados experimentalmente para diversas formas (MUNSON; YOUNG; OKIISHI, 2004). 
O arrasto na natação pode ser didaticamente dividido em três tipos, o arrasto friccional, o arrasto de forma e o arrasto de onda. O arrasto friccional possui uma relação linear com a velocidade, e representa o atrito das moléculas do fluido com o corpo que se desloca pelo fluido. Esta forma de arrasto seria influenciada pela área de superfície do corpo e pelo tamanho da camada limite (MAGLISCHO, 1999; VORONTSOV; RUMYANTSEV, 2000).

O arrasto de pressão, ou de forma possui uma relação quadrática com a velocidade, e representa a força gerada pelo diferencial de pressão a montante e a jusante do corpo que se desloca pelo meio fluido (VORONTSOV; RUMYANTSEV, 2000). Este é o tipo de arrasto predominante na natação e por isso o arrasto total é normalmente simplificado como sendo o produto entre um constante de proporcionalidade e o quadrado da velocidade de deslocamento, (k.v $\left.{ }^{2}\right)$ (AMAR, 1920). Na natação o arrasto de forma é influenciado principalmente pela orientação do corpo em relação ao escoamento (mensurado pelo $\mathrm{Cd}$ ) e ao tamanho do nadador (MAGLISCHO, 1999).

O arrasto de onda possui uma relação cúbica com a velocidade, e representa a energia cinética transmitida do corpo para o fluído e é responsável pela formação de ondas a montante (VORONTSOV; RUMYANTSEV, 2000). Na natação o arrasto de onda é influenciado principalmente por movimentos desnecessários do nadador (MAGLISCHO, 1999). Esta forma de arrasto é predominante na superfície e perde importância com o aumento da profundidade (VENNELL; PEASE; WILSON, 2006).

Tanto o arrasto total quanto as subdivisões mencionadas anteriormente podem ser avaliadas de forma passiva e ativa. O arrasto passivo é a força exercida pela água no nadador quando este não está se movendo, dentro de uma prova isso ocorre durante a fase de deslize logo após a virada ou a saída enquanto ele se encontra na posição de streamline. $\mathrm{O}$ arrasto ativo é a força exercida pela água no nadador enquanto ele se move, ou seja, enquanto ele realiza os movimentos propulsivos do nado (STAGER; TANNER, 2008).

\section{CONSIDERAÇÕES FINAIS E PERSPECTIVAS METODOLÓGICAS}

Consideramos finalmente que o atual estágio de desenvolvimento da Biomecânica é expresso pelos novos procedimentos e técnicas de investigação, nas quais reconhecemos a tendência crescente de se combinar simultaneamente várias disciplinas científicas aplicadas à análise do movimento, o que significa uma abordagem interdisciplinar aplicada ao 
relacionamento entre a Biomecânica e o Movimento humano. Assim, destacamos perspectivas metodológicas selecionadas e aplicadas à Biomecânica considerando-se tendências científicas atuais e inovativas na investigação: i) o aperfeiçoamento da técnica de movimento orientado fundamentalmente pela determinação da eficiência de movimento; ii) o aperfeiçoamento do processo de treinamento e reabilitação com base nos mecanismos indicadores da adaptação funcional e ambiental; iii) o aperfeiçoamento do mecanismo de controle de cargas internas interpretado pelo processo de otimização do rendimento; iv) o aperfeiçoamento metodológico na interação homem-máquina considerando-se os avanços da robótica e biotecnologia instrumental; e v) o aperfeiçoamento de modelos físico-matemáticos para simulação e animação gráfica do movimento, considerando-se os formalismos computacionais disponíveis.

Vivemos, na Biomecânica, um grande período analítico como ainda não observado, graças à transferência dos avanços tecnológicos aplicados à análise do movimento. Desta maneira, uma das principais tarefas da Biomecânica na projeção de perspectivas e desafios é sistematizar o conhecimento acumulado por novos conceitos, novos paradigmas, novas metodologias, novas tecnologias e novas áreas de aplicação, em particular aplicados ao Esporte.

Diante do exposto, espera-se que tenhamos tornado claro o escopo e o domínio da biomecânica para estudo do movimento humano. Como uma disciplina jovem seus métodos e protocolos são dinâmicos e estão em constante evolução, um contraste com sua fundamentação teórica, baseadas nas clássicas e bem estabelecidas leis da Física. Uma breve descrição de suas principais metodologias bem como das particularidades associadas à biomecânica do meio liquido foi apresentada, longe de objetivar uma completa descrição destes temas, mas sim permitindo um primeiro contato a partir do qual o entendimento dos experimentos biomecânicos possa ser aprimorado.

\section{REFERÊNCIAS BIBLIOGRÁFICAS}

ABDEL-AZIZ, Youssef; KARARA, Houssam; HAUCK, Michael. Direct linear transformation from comparator coordinates into object space coordinates in close-range photogrammetry. Photogrammetric engineering \& remote sensing, v. 81, n. 2, p. 103-107, fev., 2015.

AHMAD, Norhafizan e colaboradores. Reviews on various inertial measurement unit (IMU) sensor applications. International journal of signal processing systems, v. 1, n. 2, p. 256-262, 
2013.

AMADIO, Alberto Carlos e colaboradores. Introdução à biomecânica para análise do movimento humano: descrição e aplicação dos métodos de medição. Revista brasileira de fisioterapia, v. 3, n. 2, p. 41-54, 1999.

AMADIO, Alberto Carlos; DUARTE, Marcos. Fundamentos biomecânicos para a análise do movimento humano. [s.I.] Laboratorio de Biomecânica/Eefeusp, 1996.

AMADIO, Alberto Carlos; SERRÃO, Julio Cerca. Considerações sobre a biomecânica brasileira: uma análise da história acadêmico-científica da área. In: TANI, Go e colaboradores (Eds.). Celebrar a lusofonia: ensaios e estudos em desporto e educação física. Belo Horizonte, MG: Instituto Casa da Educação Física, 2012.

. Contextualização da biomecânica para a investigação do movimento: fundamentos, métodos e aplicações para análise da técnica esportiva. Revista brasileira de educação física e esporte, v. 21, p. 61-85, 2007.

A biomecânica em educação física e esporte. Revista brasileira de educação física e esporte, v. 25, n. spe, p. 15-24, dez. 2011.

AMAR, Jules. The human motor. Londoon, England: Routledge, 1920.

AMINIAN, Kamiar; NAJAFI, Bijan. Capturing human motion using body-fixed sensors: outdoor measurement and clinical applications. Computer animation and virtual worlds, v. 15, n. 2 , p. 79-94, 2004.

ANDERSON, John. Ludwig prandtl's boundary layer. Physics today, p. 42-48, dec., 2005.

ARCHIMEDES. On floating bodies, book I. In: HEATH, Thomas Little (Ed.). The works of archimedes. Cambridge, England: Cambridge University Press, 2009. p. 253-262.

BATISTA, Luiz Alberto; AMADIO, Alberto Carlos. Biomecânica do esporte. In: DACOSTA, Lamartine Pereira (Ed.). Atlas do esporte no Brasil. Rio de Janeiro: Shape Editora; Promoções Ltda, 2005.

BAUMANN, Wolfgang. Perspectives in methodology in biomechanics of sport. ISBSConference Proceedings Archive.1992

BERNOULLI, Daniel; BERNOULLI, Johann. Hydrodynamics and hydraulics. New York, USA: Dover Publications, 1968.

BLASER, W. Dreisprung. Schweizer turnen. St. Gallen: [s.n.], p. 661-664, 1971.

BORELLI, Giovanni Afonso. De motu animalium. [s.l.] Editio altera, 1685.

BURDEN, Adrian. How should we normalize electromyograms obtained from healthy 
participants? What we have learned from over 25years of research. Journal of electromyography and kinesiology, v. 20, n. 6, p. 1023-1035, 2010.

CIFREK, Mario e colaboradores. Surface EMG based muscle fatigue evaluation in biomechanics. Clinical biomechanics, v. 24, n. 4, p. 327-340, 2009.

DE LEVA, Paolo. Adjustments to zatsiorsky-seluyanov's segment inertia parameters. Journal of biomechanics, v. 29, n. 9, p. 1223-1230, set., 1996.

DIEM, Liselott; LÖCKEN, Manfred; HUMMEL, Siegfried. Sportförderung in Brasilien: bericht über die förderung des sports in der Föderativen Republik Brasilien durch die Bundesrepublik Deutschland 1963 bis 1982. [s.l.] Richarz, 1983.

GLASER, Roland. Biophysics: an introduction. [s.I.] Springer Science \& Business Media, 2012.

HANAVAN JR, Ernest. A mathematical model of the human body. [s.l: s.n.].

HATZE, Herbert. The meaning of the term 'biomechanics'. Journal of biomechanics, v. 7, n. 2, p. 189-190, mar., 1974.

A mathematical model for the computational determination of parameter values of anthropomorphic segments. Journal of biomechanics, v. 13, n. 10, p. 833-843, 1980.

HEIKKILA, Janne; SILVEN, Olli. A four-step camera calibration procedure with implicit image correction. Proceedings of IEEE Computer Society Conference on Computer Vision and Pattern Recognition. Anais... IEEE Comput. Soc, set., 1975. Disponível em: < https://ieeexplore.ieee.org/document/609468>. Acesso em 01 jun. 2021.

HERMENS, Hermie e colaboradores. Development of recommendations for SEMG sensors and sensor placement procedures. Journal of electromyography and kinesiology, v. 10, n. 5, p. 361-374, 17 out., 2000.

HOCHMUTH, Gerhard. Biomechanik sportlicher bewegungen. [s.l.] Sportverlag, 1974. v. 1.

LAUGA, Eric; BRENNER, Michael ; STONE, Howard. Microfluidics: The no-slip boundary condition. Springer handbook of experimental fluid mechanics, p. 1219-1240, 2005.

LENK, Maria. Natação. Rio de Janeiro: Melhoramentos, 1942.

MAGLISCHO, Ernest. Nadando ainda mais rápido. São Paulo: Manole, 1999.

MERLETTI, Roberto. Standards for reporting EMG data. Journal of electromyography and kinesiology, v. 9, n. 1, p. 3-4, 1999.

MEZÊNCIO, Bruno. Avaliação da ação de pernas na natação baseada no número de Strouhal. [s.l.] Universidade de São Paulo, 2017. 
MUNSON, Bruce; YOUNG, Donald; OKIISHI, Theodore. Fundamentos da mecânica dos fluidos. 4. ed. São Paulo: Edgard Blücher, 2004.

PENDERGAST, David e colaboradores. Quantitative analysis of the front crawl in men and women. Journal of applied physiology, v. 43, n. 3, p. 475-479, set., 1977.

PRANDTL, Ludwig. Über Flüssigkeitsbewegung bei sehr kleiner Reibung. Intern. Math. Kongr. Heidelberg, Auch: Gesammelte Abhandlungen. Anais...Leipzig, Deutschland: 1904.

REYNOLDS, Osborne. An experimental investigation of the circumstances which determine whether the motion of water shall be direct or sinuous, and of the law of resistance in parallel channels. Philosophical transactions of the royal society of London, v. 174, n. 0, p. 935-982, 1883.

. On the dynamical theory of incompressible viscous fluids and the determination of the criterion. Philosophical transactions of the royal society of London, v. 186, p. 123-164, 1895.

STAGER, Joel; TANNER, David. Natação. São Paulo: Manole, 2008.

STAUDENMANN, Didier e colaboradores. Methodological aspects of SEMG recordings for force estimation: a tutorial and review. Journal of electromyography and kinesiology, v. 20, n. 3, p. 375-387, 2010.

VENNELL, Ross; PEASE, Dave L.; WILSON, Barry. Wave drag on human swimmers. Journal of biomechanics, v. 39, p. 664-671, 2006.

VORONTSOV, Andrei; RUMYANTSEV, V. A. Resistive forces in swimming. In: ZATSIORSKY, Vladimir M. (Ed.). Biomechanics in sport. Oxford, England: Blackwell Science Ltd, 2000. p. 184204.

WAKELING, James. Patterns of motor recruitment can be determined using surface EMG. Journal of electromyography and kinesiology, v. 19, n. 2, p. 199-207, 2009.

YANAI, Toshimasa; WILSON, Barry. How does buoyancy influence front-crawl performance? Exploring the assumptions. Sports technology, v. 1, n. 2-3, p. 89-99, 2008.

ZHANG, Zhengyou. Flexible camera calibration by viewing a plane from unknown orientations. Proceedings of the Seventh IEEE International Conference on Computer Vision, v. 1, n. C, p. 0-7, 1999.

\section{Dados do primeiro autor:}

Email: mezencio@usp.br

Endereço: Escola de Educação Física e Esporte da Universidade de São Paulo - EEFE-USP. Avenida Professor Mello Moraes, 65, São Paulo, SP, CEP: 05508-030, Brasil.

Recebido em: 01/07/2021

Aprovado em: 14/07/2021 


\section{Como citar este artigo:}

MEZÊNCIO, Bruno; FERREIRA, Jacielle Carolina; AMADIO, Alberto Carlos. Biomecânica do movimento humano. Corpoconsciência, v. 25, n. 2, p. 87-109, mai./ ago., 2021. 